\title{
Estimation of AUC from 0 to Infinity in Serial Sacrifice Designs
}

\author{
Martin J. Wolfsegger \\ Department of Biostatistics, Baxter AG, Vienna, Austria \\ Thomas Jaki \\ Department of Statistics, University of South Carolina, Columbia
}

This article is published in the Journal of Pharmacokinetics and Pharmacodynamics, 32(5-6):757766, 2005. DOI: $10.1007 / \mathrm{s} 10928-005-0044-0$. The original publication is available at www.springerlink.com

\begin{abstract}
Nonclinical in vivo animal studies have to be completed before starting clinical studies of the pharmacokinetic behavior of a drug in humans. The drug exposure in animal studies is often measured by the area under the concentration time curve (AUC). The classical complete data design where each animal is sampled for analysis once per time point is usually only applicable for large animals. In the case of rats and mice, where blood sampling is restricted, the batch design or the serial sacrifice design need to be considered. In batch designs samples are taken more than once from each animal, but not at all time points. In serial sacrifice designs only one sample is taken from each animal. This paper presents an estimator for AUC from 0 to infinity in serial sacrifice designs, the corresponding variance and its asymptotic distribution.
\end{abstract}

Keywords: AUC; Bootstrap; Confidence Intervals; Serial sacrifice design

\section{Introduction}

Techniques to construct confidence intervals for the AUC from 0 to the last observed time point $t_{J}\left(\mathrm{AUC}_{0-t_{J}}\right)$ for the serial sacrifice design were presented in [1] and [2]. A method to construct confidence intervals for $\mathrm{AUC}_{0-t_{J}}$ for the batch design can be found in [3]. Formulas for confidence intervals for $\mathrm{AUC}_{0-t_{J}}$ which are applicable to all three types of designs are presented in [4].

In single bolus administrations it is desirable to construct a confidence interval for the AUC from 0 to infinity $\left(\mathrm{AUC}_{0-\infty}\right)$. Yuan [5] presented a method to 
construct confidence intervals for $\mathrm{AUC}_{0-\infty}$ for the serial sacrifice design under the assumption of a single exponential course of the elimination phase and that the elimination rate is a known fixed and inalterable value identical for all animals. This paper also addresses the assumption of a single exponential course of the elimination phase and that an estimate and its variance of the elimination rate are known from a different independent study. However, in practical medical research, these assumptions are hardly fulfilled.

Here, we present an estimator and its variance for $\mathrm{AUC}_{0-\infty}$ in the serial sacrifice design in which the elimination rate does not need to be known in advance. We assume only a single exponential course of the elimination phase.

\section{Area Estimation}

In a serial sacrifice design, measurements are taken at $J$ time points $t_{j}(1 \leq j \leq J)$ and at each time point $t_{j}$, blood is sampled from $n_{j}$ animals. Let $x_{i j}\left(1 \leq i \leq n_{j}\right)$ be the measured drug concentration from the $i^{t h}$ animal at time $t_{j}$. Let $E\left[x_{i j}\right]=$ $\mu_{x_{j}}$ and $V\left[x_{i j}\right]=\sigma_{x_{j}}^{2}$ be the population mean and population variance at time point $t_{j}$. To be physiological meaningful it is assumed that $\mu_{x_{j}}$ is so large compared with $\sigma_{x_{j}}^{2}$ that the range of $x_{i j}$ is effectively positive. The theoretical $\mathrm{AUC}_{0-\infty}$ can be defined as

$$
\mathrm{AUC}_{0-\infty}=\sum_{j=1}^{K} w_{j} \mu_{x_{j}}+\frac{\mu_{x_{K}}}{\mu_{\widehat{\lambda}}} \quad(K<J)
$$

where $K$ is a fixed integer and $\mu_{\widehat{\lambda}}$ is the elimination rate constant representing the slope of a single exponential function [6]. Using the linear trapezoidal rule, the weights $w_{j}$ equal

$$
\begin{aligned}
w_{1} & =\frac{1}{2}\left(t_{2}-t_{1}\right) \\
w_{j} & =\frac{1}{2}\left(t_{j+1}-t_{j-1}\right) \quad(2 \leq j \leq K-1) \\
w_{K} & =\frac{1}{2}\left(t_{K}-t_{K-1}\right) .
\end{aligned}
$$

This $\mathrm{AUC}_{0-\infty}$ can be estimated by

$$
\begin{aligned}
\widehat{\mathrm{AC}_{0-\infty}} & =\sum_{j=1}^{K} w_{j} \bar{x}_{j}+\frac{\bar{x}_{K}}{\widehat{\lambda}}-\beta \\
& =\sum_{j=1}^{K-1} w_{j} \bar{x}_{j}+\bar{x}_{K}\left(w_{K}+\frac{1}{\widehat{\lambda}}\right)-\beta
\end{aligned}
$$

where $\beta$ represent a constant for bias correction discussed later and $\bar{x}_{j}$ is the arithmetic mean at time point $t_{j}$ with $E\left[\bar{x}_{j}\right]=E\left[x_{i j}\right]=\mu_{x_{j}}$ and $V\left[\bar{x}_{j}\right]=\sigma_{\bar{x}_{j}}^{2}=$ $\sigma_{x_{j}}^{2} n_{j}^{-1}$. In addition, let $\hat{\lambda}$ be the estimate of the elimination rate constant 
which is assessed on the last $J-K$ time points and is therefore independent from $\widehat{\mathrm{AUC}_{0-}} \widehat{\mathrm{U}}_{K}$.

Our representation of the theoretical $\mathrm{AUC}_{0-\infty}$ ensures the stochastically independence between $\sum_{j=1}^{K} w_{j} \bar{x}_{j}$ and $\hat{\lambda}$ which enables the straightforward derivation of the variance for $\widehat{\mathrm{AUC}_{0-}}$.

This variance for $\mathrm{AUC}_{0-\infty}$ can be used to construct a bootstrap- $t$ confidence interval. Bootstrap- $t$ confidence intervals work well on location parameter [7] and provide better coverage properties than bootstrap percentile confidence intervals for small sample sizes [8]. Furthermore, the bootstrap percentile method is only first-order accurate whereas the bootstrap- $t$ method is second order accurate $[9]$.

\section{Elimination Rate}

The estimate $\hat{\lambda}$ for the elimination rate constant $\mu_{\widehat{\lambda}}$ can be calculated using linear regression on the last $J-K$ time points on the natural logarithmconcentration versus time curve and can be formulated as

$$
\widehat{\lambda}=(-1) \sum_{j=K+1}^{J} u_{j} \bar{y}_{j}
$$

where

$$
\begin{aligned}
& y_{i j}=\ln \left(x_{i j}\right)+\frac{1}{2} \frac{\sigma_{x_{j}}^{2}}{\mu_{x_{j}}^{2}} \\
& u_{j}=a_{j}\left(\sum_{l=K+1}^{J} a_{l}^{2}\right)^{-1} \\
& a_{j}=t_{j}-\frac{1}{J-K} \sum_{l=K+1}^{J} t_{l} .
\end{aligned}
$$

Note that $y_{i j}$ is a bias corrected representation of the log-transformed values to ensure an unbiased estimation of the elimination rate constant. The straightforward transformation using the delta method leads to $E\left[\ln \left(x_{i j}\right)\right] \approx$ $\ln \left(\mu_{x_{j}}\right)-\frac{1}{2} \sigma_{x_{j}}^{2} \mu_{x_{j}}^{-2}$ which underestimates $\ln \left(\mu_{x_{j}}\right)$ by the constant factor $\frac{1}{2} \sigma_{x_{j}}^{2} \mu_{x_{j}}^{-2}$. The expected value and variance of the bias corrected log-transformed values are

$$
\begin{aligned}
E\left[\ln \left(x_{i j}\right)+\frac{1}{2} \frac{\sigma_{x_{j}}^{2}}{\mu_{x_{j}}^{2}}\right] & =\mu_{y_{j}} \approx \ln \left(\mu_{x_{j}}\right) \\
V\left[\ln \left(x_{i j}\right)+\frac{1}{2} \frac{\sigma_{x_{j}}^{2}}{\mu_{x_{j}}^{2}}\right] & =V\left[\ln \left(x_{i j}\right)\right]=\sigma_{y_{j}}^{2} \approx \frac{\sigma_{x_{j}}^{2}}{\mu_{x_{j}}^{2}}-\frac{1}{4} \frac{\sigma_{x_{j}}^{4}}{\mu_{x_{j}}^{4}} .
\end{aligned}
$$


The arithmetic mean of the bias corrected log-transformed values at time point $t_{j}$ is denoted as $\bar{y}_{j}$ with $E\left[\bar{y}_{j}\right]=E\left[y_{i j}\right]=\mu_{y_{j}} \approx \ln \left(\mu_{x_{j}}\right)$ and $V\left[\bar{y}_{j}\right]=\sigma_{\bar{y}_{j}}^{2}=$ $\sigma_{y_{j}}^{2} n_{j}^{-1}$. The expected value and the variance of the elimination rate are

$$
\begin{aligned}
& E[\widehat{\lambda}]=\mu_{\widehat{\lambda}}=(-1) \sum_{j=K+1}^{J} u_{j} \ln \left(\mu_{x_{j}}\right) \approx(-1) \sum_{j=K+1}^{J} u_{j} \mu_{y_{j}} \\
& V[\widehat{\lambda}]=\sigma_{\widehat{\lambda}}^{2} \approx \sum_{j=K+1}^{J} u_{j}^{2}\left(\frac{\sigma_{\bar{x}_{j}}^{2}}{\mu_{x_{j}}^{2}}-\frac{1}{4} \frac{\sigma_{\bar{x}_{j}}^{4}}{\mu_{x_{j}}^{4}}\right)=\sum_{j=K+1}^{J} u_{j}^{2} \sigma_{\bar{y}_{j}}^{2} .
\end{aligned}
$$

\section{Moments of Areas}

The expected value and variance for the $\mathrm{AUC}_{\mathrm{C}_{0-t_{K-1}}}$ equal [2]

$$
\begin{aligned}
& E\left[\sum_{j=1}^{K-1} w_{j} \bar{x}_{j}\right]=\sum_{j=1}^{K-1} w_{j} \mu_{x_{j}} \\
& V\left[\sum_{j=1}^{K-1} w_{j} \bar{x}_{j}\right]=\sum_{j=1}^{K-1} w_{j}^{2} \sigma_{\bar{x}_{j}}^{2} .
\end{aligned}
$$

The expected value of the remaining area without the bias correction $\beta$ results by using the delta method in

$$
E\left[\bar{x}_{K}\left(w_{K}+\frac{1}{\widehat{\lambda}}\right)\right] \approx w_{K} \mu_{x_{K}}+\frac{\mu_{x_{K}}}{\mu_{\widehat{\lambda}}}+\frac{\mu_{x_{K}} \sigma_{\widehat{\lambda}}^{2}}{\mu_{\widehat{\lambda}}^{3}}
$$

showing a overestimation of $w_{K} \mu_{x_{K}}+\mu_{x_{K}} \mu_{\widehat{\lambda}}^{-1}$ by the constant factor $\mu_{x_{K}} \sigma_{\widehat{\lambda}}^{2} \mu_{\widehat{\lambda}}^{-3}$. Therefore we define the bias correction as

$$
\beta=\frac{\mu_{x_{K}} \sigma_{\widehat{\lambda}}^{2}}{\mu_{\widehat{\lambda}}^{3}}
$$

leading to

$$
\begin{aligned}
& E\left[\bar{x}_{K}\left(w_{K}+\frac{1}{\hat{\lambda}}\right)-\beta\right] \approx w_{K} \mu_{x_{K}}+\frac{\mu_{x_{K}}}{\mu_{\widehat{\lambda}}} \\
& V\left[\bar{x}_{K}\left(w_{K}+\frac{1}{\widehat{\lambda}}\right)-\beta\right] \approx \mu_{x_{K}}^{2}\left(\frac{\sigma_{\widehat{\lambda}}^{2}}{\mu_{\widehat{\lambda}}^{4}}\right)+\sigma_{\bar{x}_{K}}^{2}\left(\frac{\sigma_{\widehat{\lambda}}^{2}}{\mu_{\hat{\lambda}}^{4}}\right)+ \\
& \sigma_{\bar{x}_{K}}^{2}\left(w_{K}+\frac{1}{\mu_{\widehat{\lambda}}}+\frac{\sigma_{\widehat{\lambda}}^{2}}{\mu_{\hat{\lambda}}^{3}}\right)^{2}
\end{aligned}
$$


an unbiased estimate of this area. Hence the expected value and the variance for ${\mathrm{A} \widehat{\mathrm{UC}_{0-}}}_{\infty}$ are

$$
\begin{aligned}
E\left[\widehat{\mathrm{AUC_{0 } - \infty}}\right] \approx & \sum_{j=1}^{K-1} w_{j} \mu_{x_{j}}+\mu_{x_{K}}\left(w_{K}+\frac{1}{\mu_{\widehat{\lambda}}}\right)=\sum_{j=1}^{K} w_{j} \mu_{x_{j}}+\frac{\mu_{x_{K}}}{\mu_{\widehat{\lambda}}} \\
V\left[\widehat{\mathrm{AUC_{0 } - \infty}}\right] \approx & \sum_{j=1}^{K-1} w_{j}^{2} \sigma_{\bar{x}_{j}}^{2}+\left(\frac{\sigma_{\widehat{\lambda}}^{2}}{\mu_{\widehat{\lambda}}^{4}}\right)\left(\mu_{x_{K}}^{2}+\sigma_{\bar{x}_{K}}^{2}\right)+ \\
& \sigma_{\bar{x}_{K}}^{2}\left(w_{K}+\frac{1}{\mu_{\hat{\lambda}}}+\frac{\sigma_{\widehat{\lambda}}^{2}}{\mu_{\widehat{\lambda}}^{3}}\right)^{2} .
\end{aligned}
$$

\section{Variance Estimation}

The unknown variance of $\widehat{\mathrm{AUC}_{0-}}$ presented in equation 19 can be estimated by

$$
\begin{aligned}
\widehat{V}\left[\widehat{\mathrm{AUC} C_{0-}}\right]= & \sum_{j=1}^{K-1} w_{j}^{2} s_{\bar{x}_{j}}^{2}+\frac{\sum_{l=K+1}^{J} u_{l}^{2} s_{\bar{y}_{l}}^{2}}{\left(\sum_{l=K+1}^{J} u_{l} \bar{y}_{l}\right)^{4}}\left(\bar{x}_{K}^{2}+s_{\bar{x}_{K}}^{2}\right) \\
& s_{\bar{x}_{K}}^{2}\left(w_{K}-\frac{1}{\sum_{l=K+1}^{J} u_{l} \bar{y}_{l}}-\frac{\sum_{l=K+1}^{J} u_{l}^{2} s_{\bar{y}_{l}}^{2}}{\left(\sum_{l=K+1}^{J} u_{l} \bar{y}_{l}\right)^{3}}\right)^{2}
\end{aligned}
$$

where

$$
\begin{aligned}
\bar{x}_{j}=\frac{1}{n_{j}} \sum_{i=1}^{n_{j}} x_{i j} & \bar{y}_{j}=\frac{1}{n_{j}} \sum_{i=1}^{n_{j}} y_{i j} \\
s_{x_{j}}^{2}=\frac{1}{\left(n_{j}-1\right)} \sum_{i=1}^{n_{j}}\left(x_{i j}-\bar{x}_{j}\right)^{2} & s_{y_{j}}^{2}=\frac{1}{\left(n_{j}-1\right)} \sum_{i=1}^{n_{j}}\left(y_{i j}-\bar{y}_{j}\right)^{2} \\
s_{\bar{x}_{j}}^{2}=\frac{1}{n_{j}} s_{x_{j}}^{2} & s_{\bar{y}_{j}}^{2}=\frac{1}{n_{j}} s_{y_{j}}^{2} .
\end{aligned}
$$

The bias corrections in equations 5 and 15 can also be assessed by using these estimates for $\mu_{x_{j}}, \mu_{y_{j}}, \sigma_{x_{j}}^{2}, \sigma_{y_{j}}^{2}, \sigma_{\bar{x}_{j}}^{2}$ and $\sigma_{\bar{y}_{j}}^{2}$.

\section{Asymptotic Distribution}

Lemma 1 Let $\left\{t_{j}\right\} \in \mathbb{R}_{0}^{+}$be a strictly monotone increasing sequence of fixed time points with $1 \leq j \leq J$. Let $n_{j}=n$ and let the range of the $x_{i j}$ be effectively 
positive. When the Lindeberg-Levy conditions hold at every time point $t_{j}$, then

$$
\sqrt{n}\left(\sum_{j=1}^{K} w_{j} \bar{x}_{j}+\frac{\bar{x}_{K}}{\widehat{\lambda}}-\left(\sum_{j=1}^{K} w_{j} \mu_{x_{j}}+\frac{\mu_{x_{K}}}{\mu_{\widehat{\lambda}}}\right)\right) \stackrel{d}{\rightarrow} N\left(0, \xi^{2}\right) \quad(K<J)
$$

where

$$
\xi^{2}=\sum_{j=1}^{K-1} w_{j}^{2} \sigma_{x_{j}}^{2}+\sigma_{x_{K}}^{2}\left(w_{K}+\frac{1}{\mu_{\widehat{\lambda}}}\right)^{2}+\frac{\mu_{x_{K}}^{2}}{\mu_{\widehat{\lambda}}^{4}} \sum_{j=K+1}^{J} u_{j}^{2} \sigma_{y_{j}}^{2} .
$$

Proof. Let $\boldsymbol{T}_{n}=\left(\bar{x}_{1}, \ldots, \bar{x}_{K}, \bar{y}_{K+1}, \ldots, \bar{y}_{J}\right)$ with pairwise independent elements, and let $E\left[\boldsymbol{T}_{n}\right]=\boldsymbol{\mu}=\left(\mu_{x_{1}}, \ldots, \mu_{x_{K}}, \ln \left(\mu_{x_{K+1}}\right), \ldots, \ln \left(\mu_{x_{J}}\right)\right)$ and $V\left[\boldsymbol{T}_{n}\right]=$ $\frac{1}{n} \Sigma$. By the multivariate central limit theorem the sequence $\sqrt{n}\left(\boldsymbol{T}_{n}-\boldsymbol{\mu}\right)$ converges in distribution to $N(\mathbf{0}, \Sigma)$. Let $\phi: \mathbb{R}^{J} \rightarrow \mathbb{R}^{1}$ be defined as

$$
\begin{aligned}
\phi\left(\bar{x}_{1}, \ldots, \bar{x}_{K}, \bar{y}_{K+1}, \ldots, \bar{y}_{J}\right) & =\sum_{j=1}^{K-1} w_{j} \bar{x}_{j}+\bar{x}_{K}\left(w_{K}+\frac{1}{\hat{\lambda}}\right) \\
& =\sum_{j=1}^{K-1} w_{j} \bar{x}_{j}+\bar{x}_{K}\left(w_{K}-\left(\sum_{j=K+1}^{J} u_{j} \bar{y}_{j}\right)^{-1}\right)
\end{aligned}
$$

with the partial derivatives at $\boldsymbol{\mu}$

$$
\phi_{\boldsymbol{\mu}}^{\prime}=\left(w_{1}, \ldots, w_{K-1}, w_{K}+\frac{1}{\mu_{\widehat{\lambda}}}, \frac{\mu_{x_{K}} u_{K+1}}{\mu_{\widehat{\lambda}}^{2}}, \ldots, \frac{\mu_{x_{K}} u_{J}}{\mu_{\widehat{\lambda}}^{2}}\right) .
$$

Since $\left\{t_{j}\right\}$ is strictly monotone increasing and $\mu_{x_{j}}>0$ results in $\mu_{\hat{\lambda}}>0$. All elements of $\phi_{\boldsymbol{\mu}}^{\prime}$ are therefore continuous at $\boldsymbol{\mu}$ and the sequence $Z_{n}=\sqrt{n}\left(\phi\left(\boldsymbol{T}_{n}\right)-\phi(\boldsymbol{\mu})\right)$ converges in distribution to $N\left(\mathbf{0}, \phi_{\boldsymbol{\mu}}^{\prime} \Sigma\left(\phi_{\boldsymbol{\mu}}^{\prime}\right)^{t}\right)$ by the multivariate delta method which is a consequence of Taylor's theorem and Slutky's theorems [10] with

$$
\phi_{\boldsymbol{\mu}}^{\prime} \Sigma\left(\phi_{\boldsymbol{\mu}}^{\prime}\right)^{t}=\sum_{j=1}^{K-1} w_{j}^{2} \sigma_{x_{j}}^{2}+\sigma_{x_{K}}^{2}\left(w_{K}+\frac{1}{\mu_{\widehat{\lambda}}}\right)^{2}+\frac{\mu_{x_{K}}^{2}}{\mu_{\widehat{\lambda}}^{4}} \sum_{j=K+1}^{J} u_{j}^{2} \sigma_{y_{j}}^{2} .
$$

Note that under the Lindeberg-Levy conditions

$$
\lim _{n \rightarrow \infty} \beta=\lim _{n \rightarrow \infty} \frac{\mu_{x_{K}}}{\mu_{\hat{\lambda}}^{3}} \sum_{j=K+1}^{J} u_{j}^{2} \frac{\sigma_{y_{j}}^{2}}{n}=0
$$

and $\sum_{j=1}^{K} w_{j} \bar{x}_{j}+\bar{x}_{K} \widehat{\lambda}^{-1}$ is therefore an asymptotically unbiased estimator for $\sum_{j=1}^{K} w_{j} \mu_{x_{j}}+\mu_{x_{K}} \mu_{\hat{\lambda}}^{-1}$ as shown in the Lemma. 


\section{Confidence Intervals}

A large sample approximation of the $1-\alpha$ confidence interval for $\mathrm{AUC}_{0-\infty}$ equals

$$
\left[\mathrm{A} \widehat{\mathrm{UC}_{0-} \infty}-z_{1-\frac{\alpha}{2}} \frac{\xi}{\sqrt{n}} ; \widehat{\mathrm{AUC_{0- }}}+z_{1-\frac{\alpha}{2}} \frac{\xi}{\sqrt{n}}\right]
$$

where $z_{1-\frac{\alpha}{2}}$ is the $1-\frac{\alpha}{2}$ quantile of a standard normal distribution. This confidence interval depends on the unknown parameter $\xi$ which can be regarded as a function of $\mu_{x_{j}}, \mu_{y_{j}}, \sigma_{x_{j}}^{2}$ and $\sigma_{y_{j}}^{2}$. In practice, this unknown variance can be assessed by using the corresponding estimates presented in equations 21 and 22 .

Using the variance estimator presented in equation 20 leads to a bootstrap- $t$ confidence interval without using the inefficient nested bootstrap. The following bootstrap- $t$ confidence interval at level $1-\alpha$ is suggested for small sample sizes

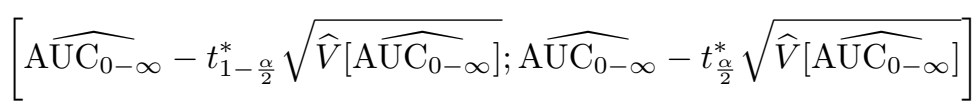

where $t_{\frac{\alpha}{2}}^{*}$ and $t_{1-\frac{\alpha}{2}}^{*}$ are the corresponding quantiles of the bootstrap distribution of the pivot statistic. It is clear that stratified bootstrapping has to be performed using the different time points as strata variables.

\section{Simulations}

We use the following very simple and artificial concentration data model

$$
x=f(t)=\left\{\begin{array}{cc}
c^{-1} a t & t \leq c \\
a \exp (-b(t-c)) & t \geq c
\end{array}\right.
$$

which leads to

$$
\mathrm{AUC}_{0-\infty}=\frac{a}{c} \int_{0}^{c} t d t+a \int_{c}^{\infty} \exp (-b(t-c)) d t .
$$

To take care of the bias of the linear trapezoidal rule to approximate the integral, the true $\mathrm{AUC}_{0-\infty}$ was defined by equation 1 on $\mu_{x_{j}}=f\left(t / t=t_{j}\right)$ specified at baseline and at 10 fixed time points which is a typical number of time points in a serial sacrifice design.

Empirical coverage probabilities of the asymptotic and bootstrap- $t$ confidence intervals were assessed by simulations. Within each simulation run, $n$ random samples were drawn from the different time points using normal and log-normal distributed errors. We used a per time point variability of $20 \%$ for normal distributed errors and $5 \%$ for log-normal distributed errors (on the $\log$-scale).

For the bootstrap- $t$ confidence intervals, 1000 bootstrap replications were used. The empirical coverage probability are based on 5000 simulation runs 
and a nominal coverage probability of $1-\alpha=0.95$. The elimination rate was calculated based on the last 3 time points. Source code in R [11] for calculation of bootstrap- $t$ and asymptotic confidence intervals can be obtained from the authors.

\section{Results}

Table 1: Empirical Coverage Using a Nominal Coverage Probability of $95 \%$

\begin{tabular}{lccc}
\hline Error distribution & $\begin{array}{c}\text { Sample size } \\
\text { per time point }\end{array}$ & \multicolumn{2}{c}{ Type of confidence Interval } \\
Asymptotic & Bootstrap- $t$ \\
\hline Normal & 3 & 0.782 & 0.862 \\
& 5 & 0.841 & 0.893 \\
& 100 & 0.952 & 0.936 \\
Log-normal & & & \\
& 3 & 0.859 & 0.913 \\
& 5 & 0.895 & 0.911 \\
& 100 & 0.942 & 0.948 \\
\hline
\end{tabular}

Simulations using sample size of 3 and 5 per time point which are typical sample sizes for this type of studies indicate better coverage probabilities using bootstrap- $t$ confidence intervals for normal and log-normal distributed errors. Asymptotic confidence intervals based on the normal distribution are therefore not recommended for such small sample size due to the substantial lack of coverage. Using a theoretical sample size of 100 animals per time point, both the asymptotic and the bootstrap- $t$ confidence intervals indicate sufficient coverage.

For asymmetrically distributed statistics like $\widehat{\mathrm{AUC}_{0-}}$ the bootstrap- $t$ sometimes performs poorly [12]. However, the bootstrap- $t$ confidence interval indicate better coverage than the confidence interval based on the asymptotic normal distribution for a small sample size per time point.

\section{Example}

The female mice data [5] are used to calculate a $95 \%$ bootstrap- $t$ confidence interval for $\mathrm{AUC}_{0-\infty}$ using 1000 bootstrap replications per time point. The elimination rate is calculated using data from the last three time points. The

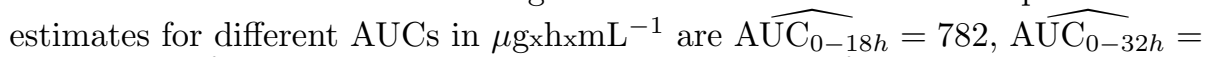
959 and $\widehat{\mathrm{AUC}_{0-}}=1030$ where $\widehat{\beta}=6.27$ and $\widehat{V}\left[\widehat{\mathrm{AUC}_{0-\infty}}\right]=72.1^{2}$. Finally, the $95 \%$ bootstrap- $t$ confidence interval for $\mathrm{AUC}_{0-\infty}$ ranges from 832 to 1233 $\mu \mathrm{g}_{\mathrm{xh}} \mathrm{mL} \mathrm{L}^{-1}$ whereas the $95 \%$ confidence interval using the asymptotic normal distribution ranges from 890 to $1171 \mu \mathrm{g}_{\mathrm{xh}} \mathrm{mL} \mathrm{L}^{-1}$. 


\section{Discussion}

The presented estimator and its variance depend heavily on the values observed at time point $t_{K}$. The time point $t_{K}$ and the subsequent time points used to calculate the elimination rate should be therefore chosen with care. We suggest to chose $t_{K}$ to be greater than the minimum time to reach the maximum concentration $\left(\mathrm{T}_{\max }\right)$.

Assuming identical variability at specific time points, the variance of the elimination rate decrease as the sum $\sum_{l=K+1}^{J} u_{l}^{2}$ decrease. To minimize the variance of the elimination rate $\sigma_{\widehat{\lambda}}^{2}$ the time points on which these parameter is estimated should be a long way away from each other.

In this article, we made no assumption on the error distribution and from the theoretical point of view both confidence intervals presented are only asymptotically of level $1-\alpha$. It would therefore be desirable to develop the exact distribution of the presented estimate under assumption of normal and log-normal distributed errors. However, the bootstrap- $t$ confidence interval indicate better coverage probabilities for small sample sizes than the confidence interval based on the asymptotic normal distribution.

We assume that the availability of the covariance of $\widehat{\mathrm{UCC}_{0-}}{ }_{J}$ and the elimination rate leads to an improved estimate and variance of $\mathrm{AUC}_{0-\infty}$, because data points can be used twice. This covariance is subject to further research.

Acknowledgement The authors would like to thank the Journal of Pharmacokinetics and Pharmacodynamics for publishing this article and giving helpful comments during the process of writing it.

\section{References}

[1] A. J. Bailer. Testing for the equality of area under the curves when using destructive measurement techniques. Journal of Pharmacokinetics and Biopharmaceutics 16(3):303-309 (1988).

[2] J. R. Nedelman, E. Gibiansky, D. T. W. Lau. Applying Bailer's method for AUC confidence intervals to sparse sampling. Pharmaceutical Research 12(1):124-128 (1995).

[3] J. Singer and M. Berger. The Jackknife applied to incomplete blood sampling models. Pharmaceutical Statistics 2(3):161-166 (2003).

[4] D. J. Holder, F. Hsuan, R. Dixit, K. Soper. A method for estimating and testing area under the curve in serial sacrifice, batch, and complete data designs. Journal of Biopharmaceutical Statistics 9(3):451-464 (1999).

[5] J. Yuan. Estimation of variance for AUC in animal studies. Journal of Pharmaceutical Sciences 82(7):761-763 (1993). 
[6] M. Gibaldi and D. Perrier. Pharmacokinetics, 2nd edition. Marcel Dekker, New York, page 411 (1982).

[7] B. Efron and R. J. Tibshirani. An Introduction to the Bootstrap. Chapman and Hall, London, page 326 (1993).

[8] J. Shao and D. Tu. The Jackknife and Bootstrap. Springer, New York, page 151 (1996).

[9] A. C. Davison and D. V. Hinkley. Bootstrap Methods and their Applications. Cambridge Series in Statistical and Probabilistic Mathematics. Cambridge University Press, page 212 (1999).

[10] A. W. Van der Vaart. Asymptotic Statistics. Cambridge University Press, United Kingdom, page 26 (1998).

[11] R Development Core Team. R: A language and environment for statistical computing. R Foundation for Statistical Computing. Vienna, Austria (2004).

[12] M. Ploner. Bootstrap confidence intervals - an overview and comparisons by means of selected applications. Ph.D. dissertation, University of Vienna (2002). 